\title{
Energy Absorption Behavior of Polyurea Under Laser-Induced Dynamic Mixed-Mode Loading
}

\author{
Kailash C. Jajam ${ }^{1,2} \cdot$ Nancy R. Sottos ${ }^{1,2}$
}

Received: 24 March 2016/ Accepted: 26 May 2016/Published online: 7 June 2016

(c) Society for Experimental Mechanics, Inc 2016

\begin{abstract}
The energy absorption response of polyurea thin films is investigated under laser-induced dynamic mixedmode loading. By exploiting the substrate geometry, the arrival times and the convenience of two simultaneously detected waves (longitudinal and shear), the transient shear loading of thin films is achieved under mixed-mode conditions. Polyurea films are fabricated on fused silica prismatic $\left(90^{\circ}-45^{\circ}-45^{\circ}\right)$ substrates by spin coating a polyurea mixture diluted with tetrahydrofuran solvent. A high amplitude shear wave loading of the polyurea films is attained by the mode-conversion of a laser generated compressive stress wave at an oblique face of the prism substrate. Mixed-mode dynamic shear experiments are conducted on $100 \mathrm{~nm}$ thick aluminum and $2 \mu \mathrm{m}$ thick polyurea films over a range of laser loading fluences (energy/unit area). The free surface displacement, surface velocity and energy fluence histories are inferred from the shear portion of high-speed interferometric measurements. On comparing input-output velocities and fluence parameters, test results show velocity retardation and significant energy absorption (50-65\%) by the polyurea films. Postmortem spallation profiles reveal film wrinkling, tearing
\end{abstract}

Nancy R. Sottos

n-sottos@illinois.edu

Kailash C. Jajam

kcjajam@illinois.edu

1 Department of Materials Science and Engineering, University of Illinois at Urbana-Champaign, 1304 West Green Street, Urbana, IL 61801, USA

2 Beckman Institute for Advanced Science and Technology, University of Illinois at Urbana-Champaign, 405 North Mathews Avenue, Urbana, IL 61801, USA and a directional failure pattern, indicating shear as the dominant failure mode under the mixed-mode conditions.

Keywords Thin film · Polyurea · Energy absorption · Mixed-mode loading · Dynamic failure - Laser spallation · Optical metrology

\section{Introduction}

Continued demand for safety and survival against blast, ballistic impacts, and high-speed collisions routinely requires high energy absorbing layered/laminated architectures (e.g., helmet lining, laminated glass, body armor, laser shields, interlayer sandwich panels, etc.). In such scenarios, polymers offer significant advantages over conventional materials due to their low density, ease of processing, cost-effectiveness, optical transparency, energy absorbing and vibration damping properties. In the last few years, polyurea, a class of segmented block copolymer has drawn increasing attention for its energy absorbing and shock wave attenuation/dispersion characteristics in coating/liner or interlayer for blast-tolerant sandwich panels and structures.

The viscoelastic rate sensitive stress-strain behavior of polyurea has been studied by several researchers [1-5]. Mock et al. [5] performed a series of plate impact experiments in order to evaluate dynamic properties of polyurea from the measured shock velocity-particle velocity Hugoniot relationship. The uniaxial tensile response of polyurea at intermediate strain rates $\left(0.06-573 \mathrm{~s}^{-1}\right)$ was investigated by Roland et al. [6] using a drop weight test instrument. Sarva et al. [7], Shim and Mohr [8] conducted uniaxial quasi-static and split-Hopkinson-pressure-bar (SHPB) compression studies on polyurea over a range of strain 
rates $\left(10^{-3}-10^{4} \mathrm{~s}^{-1}\right)$. A number of studies have also been carried out to investigate blast resistance of the polyurea based layered structures. For example, in their shock tube experiments Tekalur et al. [9] and Gardner et al. [10] observed improved blast resistance of the composite sandwich panels with a polyurea interlayer, compared to the no interlayer cases. The dynamic stress-strain response of an $1 \mathrm{~mm}$ thick polyurea layer sandwiched between two acrylic plates was studied by Youssef and Gupta [11] using laser generated stress waves under tensile mode at a strain rate of $\sim 10^{5} \mathrm{~s}^{-1}$. Amini et al. [12] investigated the effect of the relative position of the polyurea layer with respect to the loading direction on steel-polyurea bilayer plates. They noted enhanced energy absorption when the polyurea is layered on the back face relative to the load-receiving side compared to the front face. Roland et al. [13] and Xue et al. [14] noted improved ballistic penetration resistance on steel plates with polyurea coating. A more recent study by Jajam et al. $[15,16]$ on impact behavior of polyurethane based interpenetrating polymer networks (IPNs) reveals a 3-4 fold increase in energy absorption capacity of IPNs relative to neat PMMA when subjected to low-velocity impacts using a drop tower apparatus. Among the numerical works, a study by Bahei-El-Din et al. [17] find that a thin polyurea interlayer between the face sheet and the foam core of a sandwich composite is beneficial in blast mitigation. Computational studies by Grujicic et al. $[18,19]$ show improved blast and shock wave mitigation capabilities of polyurea relative to the conventional foam when used as a suspension pad/liner in helmets.

Most of the dynamic property characterization of polyurea is limited to tensile, SHPB compression or shock tube loading experiments with strain rates on the order of $10^{2}$ and $10^{4} \mathrm{~s}^{-1}$, respectively. In contrast to the aforementioned loadings, the laser spallation techniques [20-27] employ laser-generated high amplitude acoustic stress pulses of sharp temporal rise and fall times (a few nanosecond) to dynamically load a thin film to ultrahigh strain rates of the order $10^{7} \mathrm{~s}^{-1}$. Guided by parametric studies on laser tensile loading, Wang et al. [25, 28] successfully modified the laser spallation technique to achieve dynamic mixed-mode loading of thin films by allowing the initial longitudinal stress pulse to mode convert at an oblique surface of a fused silica triangular prism into a high amplitude shear wave and a secondary longitudinal wave. In the current work, we employ this novel method to dynamically load the polyurea thin films under mixed-mode conditions.

While majority of previous works on polyurea involved uniaxial (tensile/compression) normal loading, in most practical applications, layered interfaces tend to fail under mixed-mode conditions [29] due to the cumulative effect of both normal and in-plane shear components at the interface. Much of the published research to date on polyurea deals with blast mitigation and SHPB studies, and very few describe energy absorption properties. Further, the reported ones are mostly qualitative and have been studied at intermediate strain rates $\left(10^{2}-10^{4} \mathrm{~s}^{-1}\right)$ under longitudinal loading. No study on dynamic mixed-mode loading of polyurea thin films has yet been documented at strain rates of the order $\sim 10^{7} \mathrm{~s}^{-1}$.

In this work, we investigate the energy absorption response of polyurea thin films subjected to laser-induced dynamic mixed-mode loading over a range of laser fluences (energy/unit area). In the following sections, the details of specimen geometry and sample preparation protocols are provided. Experimental observations include free surface displacement, surface velocity, fluence histories and typical thin film damage patterns varying with laser loading fluences. Finally, the major findings of the current study are discussed and summarized.

\section{Materials and Sample Preparation}

The substrates for laser-induced mixed-mode loading experiments consisted of fused silica (Corning 7980-2G type, IOS Optics Inc.) right-angled prisms (dimension: $3 \times 3 \times 20 \mathrm{~mm}$ ) in $90^{\circ}-45^{\circ}-45^{\circ}$ configuration as shown in Figs. 1 and 2a. The choice of fused silica ${ }^{1}$ as a substrate material was due to its nonlinear elastic characteristics, low coefficient of thermal expansion and high temperature stability. Aluminum (99.99 \% purity, Kurt J. Lesker Co.) was chosen for energy absorbing layer as well as a reflective coating because of its high thermal expansion, high reflectance (for $532 \mathrm{~nm}$ diagnostic beam wavelength) and efficiency to produce the strongest and the shortest rise-time stress pulses [33, 34]. A sodium silicate waterglass solution (Fisher Scientific) was used for the confinement of aluminum absorbing layer. An oligomeric diamine (Versalink P-1000, Air Products and Chemicals, Inc.) and a diphenylmethane diisocyanate (Isonate 143-L, Dow Chemical) were used for polyurea synthesis. Tetrahydrofuran (THF) (Fisher Scientific) was employed as a solvent to dilute poluyrea for thin film fabrication. Some of the relevant properties of materials used in this study are listed in Table 1. In order to hold the prism substrates flat while film deposition, a silicone ealstomer, polydimethylsiloxane (PDMS) (Sylgard 184, Dow Corning Corp.), was used to cast individual molds for holding the samples. Fig. $2 b$ and $c$ show the cross-sections of the two molds designed for the current experiments. The mold in Fig. $2 b$

\footnotetext{
1 With its unique negative nonlinear elasticity, fused silica softens under modest compression and helps to develop a decompression shock in the substrate and enhances the loading at the interface [30-32].
} 
Fig. 1 Schematic of laser loading of a right triangular $90^{\circ}-45^{\circ}-45^{\circ}$ fused silica prism specimen showing configuration of shear $(S)$ wave generation under dynamic mixed-mode loading

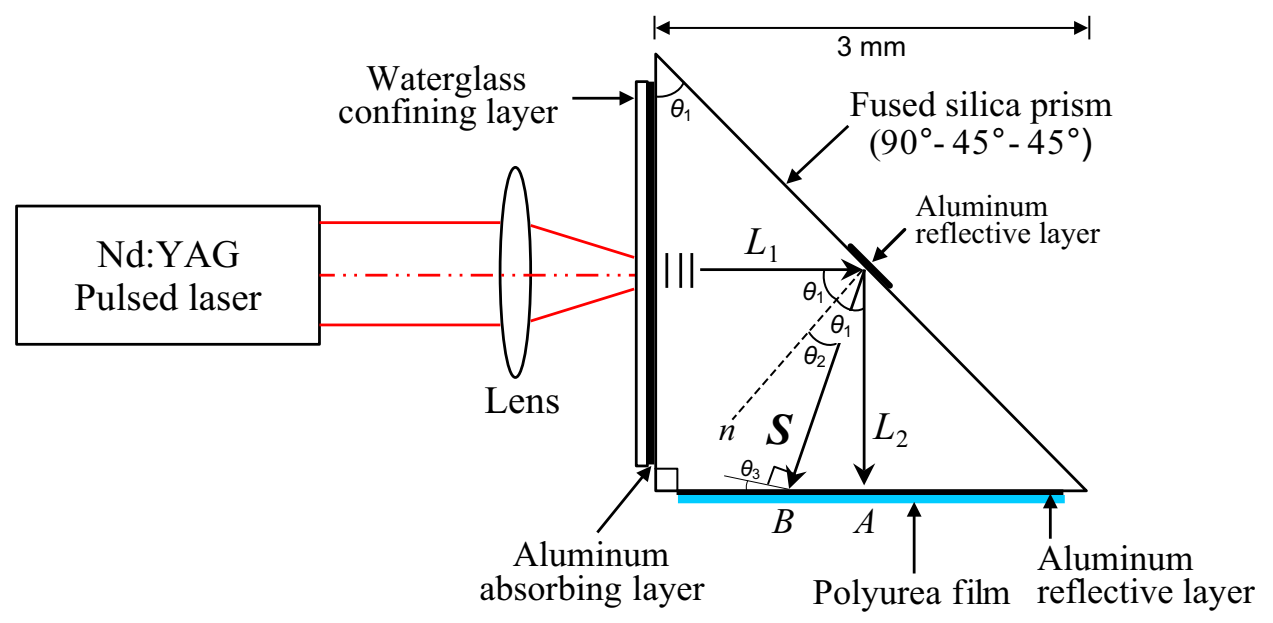

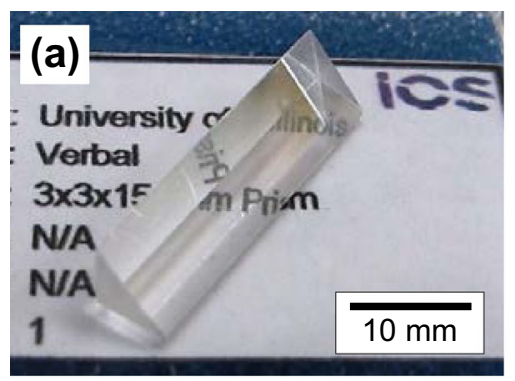
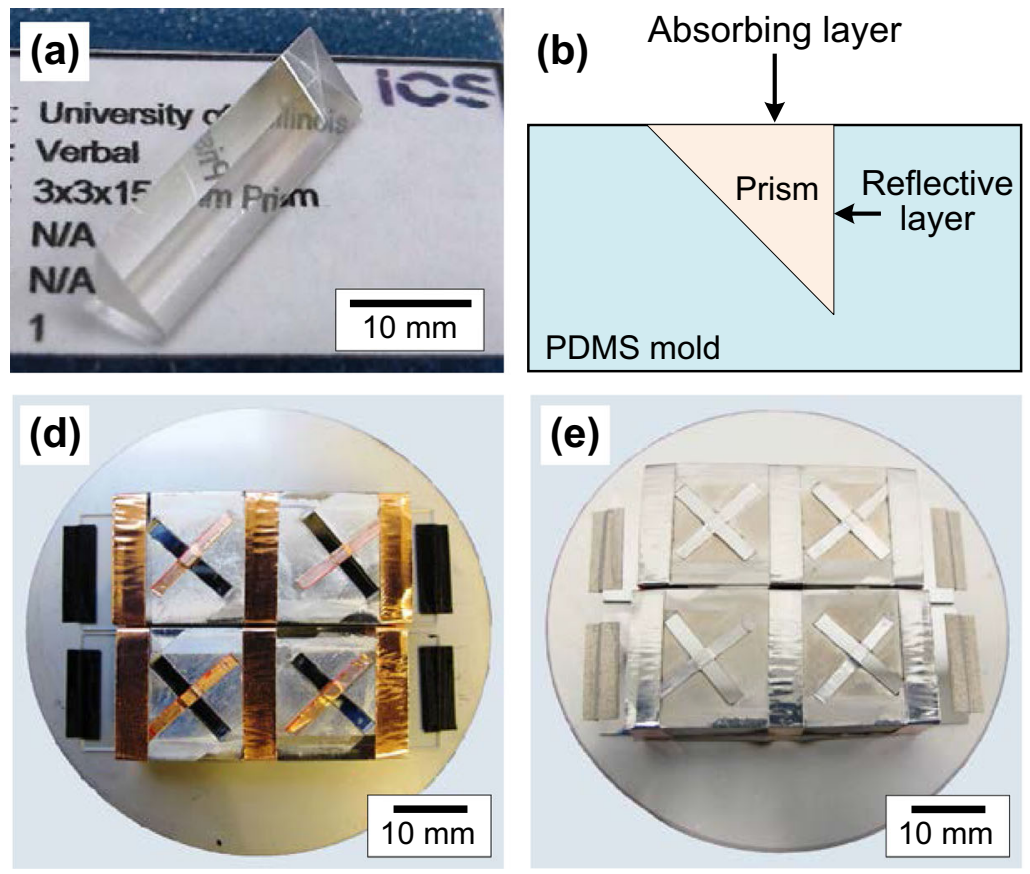
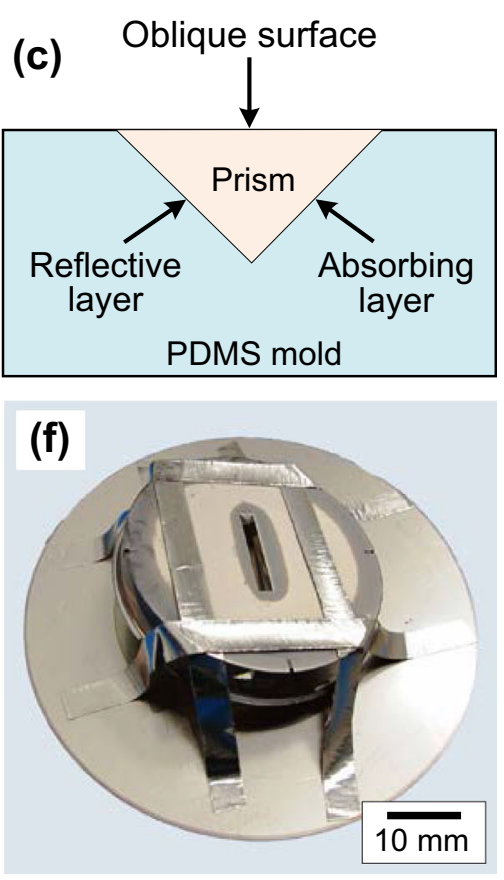

Fig. 2 Specimen fabrication for dynamic shear loading of thin films. a A right-angled $90^{\circ}-45^{\circ}-45^{\circ}$ fused silica prism $(3 \times 3 \times 20 \mathrm{~mm})$ substrate. $\mathbf{b}$ and $\mathbf{c}$ Cross section of the PDMS mold configurations for prism substrate for e-beam evaporation of aluminum on orthogonal and oblique surfaces, respectively. d Prism substrates in PDMS molds before e-beam evaporation. e Prism substrates in PDMS molds after aluminum film deposition. f PDMS mold for spin coating of waterglass layer and polyurea films

Table 1 Relevant material properties

\begin{tabular}{llll}
\hline Material & $\begin{array}{l}\text { Density, } \rho \\
\left(\mathrm{kg} / \mathrm{m}^{3}\right)\end{array}$ & $\begin{array}{l}\text { Longitudinal wave } \\
\text { speed, } C_{L}(\mathrm{~m} / \mathrm{s})\end{array}$ & $\begin{array}{l}\text { Shear wave } \\
\text { speed, } C_{S}(\mathrm{~m} / \mathrm{s})\end{array}$ \\
\hline Fused silica [25] & 2200 & 5940 & 3750 \\
Aluminum [35] & 2700 & 6370 & 3120 \\
Polyurea [5] & 950 & 1660 & 960 \\
\hline
\end{tabular}

was prepared to hold the prism while aluminum evaporation on either of the orthogonal surfaces (absorbing layer or reflective layer), whereas the configuration of mold shown in Fig. 2c was created to hold the prism when evaporating aluminum on the oblique surface to facilitate the laser alignment. 
For thin film fabrication on prism substrates, the fused silica prisms were cleaned in a piranha solution $\left(3 \mathrm{H}_{2} \mathrm{SO}_{4}: 1\right.$ $\mathrm{H}_{2} \mathrm{O}_{2}$, by volume) at $120{ }^{\circ} \mathrm{C}$ for $60 \mathrm{~min}$ to remove any organic residues off substrates. The freshly cleaned and oxidized substrates were thoroughly rinsed with deionzed water (millipore water purification unit), dried under a stream of air, and further dried in an oven at $100{ }^{\circ} \mathrm{C}$ for 15 min. Next, as shown in Figure 2d, the prisms samples were flushed into the mold cavities and a copper tape (Ted Pella Inc.) was used to mask any areas on the prisms where the film deposition was not desired for the purpose of laser alignment. An aluminum energy absorbing layer $(400 \mathrm{~nm}$ thick) and reflective/test film (100 nm thick) were deposited on vertical and horizontal orthogonal surfaces (see Fig. 1) of the prism, respectively, via electron-beam (Temescal) evaporation at a rate of 1.5-2.0 $\AA$ /s under the vacuum pressure of $2.0 \times 10^{-6}$ Torr. Fig. $2 \mathrm{~d}$ and e show fused silica prism substrates embedded in PDMS molds before and after e-beam evaporation of aluminum, respectively. A relatively large circular PDMS mold (Fig. 2f) was used to minimize edge effects while spin coating of polyurea or waterglass on the aluminum deposited orthogonal surfaces of the prisms due to their high aspect ratio (20:3). Prior to spin coating, the molds were subjected to e-beam evaporation of aluminum (100 nm thick) to facilitate surface compatibility between the prism and the mold surfaces in order to achieve a uniform deposition of polyurea or waterglass layer. Polyurea was synthesized by mixing oligomeric diamine and diphenylmethane diisocyanate in $4: 1$ ratio (by weight) and the mixture was diluted appropriately using THF. Polyurea thin films ( $2 \mu \mathrm{m}$ thick) were prepared by spin coating a solution of polyurea/THF (1:8 by weight) at $4000 \mathrm{rpm}$ on top of the aluminum (100 nm) coated orthogonal surface of the prism (see Fig. 1). Polyurea film samples were allowed to cure at ambient conditions for $24 \mathrm{~h}$ followed by additional curing at $80{ }^{\circ} \mathrm{C}$ in an oven for $24 \mathrm{~h}$. Polyurea film thickness was found to be $2.0 \pm 0.1 \mu \mathrm{m}$ as measured by stylus profilometry (P6 KLA Tencor). Finally, a water glass solution was spin coated on the energy absorbing layer, achieving a thickness of $10 \mu \mathrm{m}$ and was allowed to dry at room temperature for $1 \mathrm{~h}$ prior to testing. Two types of test films were investigated, $100 \mathrm{~nm}$ thick aluminum film for benchmark/calibration experiments and $2 \mu \mathrm{m}$ thick polyurea films fabricated on top of the $100 \mathrm{~nm}$ thick aluminum film (a reflective coating for the Michelson interferometer).

For interferometric measurements, the surface quality of polyurea thin films fabricated in the current study was assessed using a stylus surface profilometer (P6 KLA Tencor) and compared with those of bare fused silica substrate and e-beam evaporated aluminum film. The surface roughness measurements were performed on bare fused silica substrate, $100 \mathrm{~nm}$ thick aluminum and $2 \mu \mathrm{m}$ thick polyurea films over a scan length of $5 \mathrm{~mm}$ at 3-4 different locations in each sample. The digitized data obtained from the surface profiler was processed to calculate root mean square surface roughness $\left(R_{r m s}\right)$. The $R_{r m s}$ values were $2.84,3.88$ and $4.36 \mathrm{~nm}$ for bare fused silica substrate, $100 \mathrm{~nm}$ thick aluminum and $2 \mu \mathrm{m}$ thick polyurea films, respectively. The e-beam evaporated aluminum and the spin coated polyurea showed a surface waviness as small as few nanometers in height, with lateral separations of less than $1 \mu \mathrm{m}$. The $R_{r m s}$ values as well as the peak amplitudes of surface elevations in each case were smaller than the wave length of the diagnostic beam $(532 \mathrm{~nm})$ for the Michelson interferometer used in this study. Thus, from the perspective of optical metrology [36], the surface quality of e-beam evaporated aluminum and spin coated polyurea thin films is in agreement with the requirements of specular finish in order to facilitate in situ interferometric data collection in the current work.

\section{Experimental Methodology}

\section{Working Principle}

The dynamic mixed-mode loading of polyurea thin films was achieved using a right triangular $\left(90^{\circ}-45^{\circ}-45^{\circ}\right)$ prism substrate. The cross-sectional view of the substrate describing the working principle of the laser spallation technique for the shear wave loading of thin films in a mixed-mode setting is shown in Fig. 1. Stress waves are generated by impinging a high powered laser pulse on an energy absorbing material (aluminum) sandwiched between the confining layer and the substrate, causing rapid vaporization of aluminum into plasma. Because of the volumetric expansion a large pressure is created, generating a compressive stress wave, $L_{1}$, that propagates towards the oblique surface. Upon reflection at the oblique surface, stress wave, $L_{1}$ is mode-converted into a secondary longitudinal wave, $L_{2}$, and a high-amplitude shear wave, $S$. The mode-converted reflected waves $L_{2}$ and $S$ are then incident on the bottom orthogonal surface of the prism at points $A$ and $B$, respectively.

\section{Optical Setup and Laser Alignment}

The schematic and a photograph of the optical setup used for dynamic mixed-mode loading of thin films are shown in Figs. 3 and 4, respectively. The setup consisted of a Q-switched Nd:YAG infrared laser (New Wave Research Inc., Tempest $10 \mathrm{~Hz}, \lambda=1064 \mathrm{~nm}$ ) with $5 \mathrm{~ns}$ duration Gaussian pulse of continuously adjustable energy content between 0-200 $\mathrm{mJ}$ as the energy source to generate transient loading on the specimen. A Michelson interferometer 


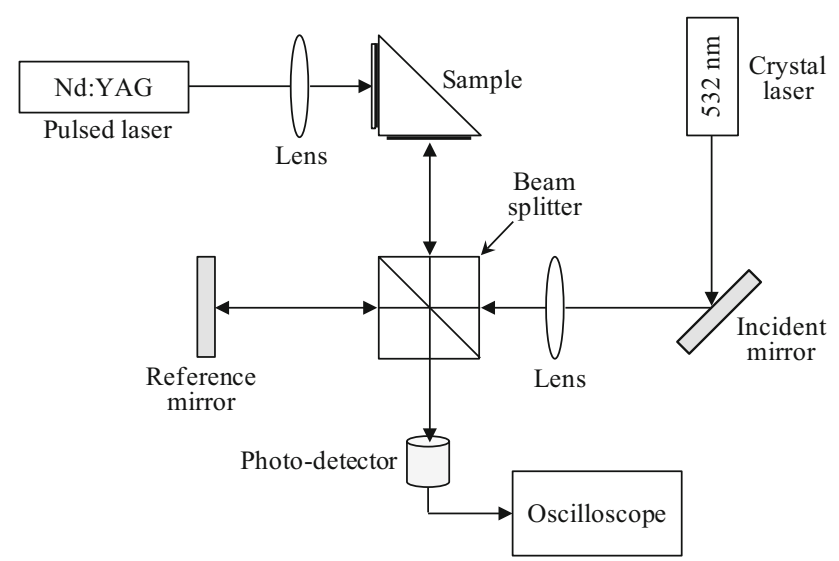

Fig. 3 Schematic of the Michelson interferometric setup for the measurement of free surface displacements under laser-induced dynamic loading of thin films

was employed to monitor the surface displacement of the thin films in real-time. The probe beam (CrystaLaser LC, $100 \mathrm{~mW}, \lambda=532 \mathrm{~nm}$ ) of the interferometer was focused at point $B$ (Fig. 1) in order to record mixed-mode interference intensity profile in terms of voltage using an ultrafast photo-detector (Electro-Optics Technology Inc., ET-2030, rise time $=300 \mathrm{ps}$ ) in conjunction with a highspeed digital oscilloscope (LeCroy LC584 A, $40 \mathrm{GHz}$ ). Care was exercised in aligning the optics so that that the beam was focused perpendicular to the film surface to the smallest possible focal spot $(\sim 100 \mu \mathrm{m})$ at the point of detection. The optical arrangement was such that 50/50 parts of the probe beam were directed normal to the surfaces of a stationary reference mirror and a sample using a beam splitting cube, and the reflected light intensity from both surfaces recombine to produce an interference signal (due to any out-of-plane motion of the sample surface) being detected by the photo-detector.

Due to the small specimen size, the transient nature of loading and the complicated stress wave mode-conversion at the oblique surface, alignment of the two lasers is critical to acquire the expected interference signal at the point of interest. A photograph shown in Fig. 5 depicts the scheme of laser alignment for mixed-mode loading experiments. A right-angled fused silica prism $(3 \times 3 \times 20 \mathrm{~mm})$ was securely held on a 3-d micro-mechanical stage. In the middle of the $20 \mathrm{~mm}$ long and $3 \mathrm{~mm}$ wide prism, a narrow section of $2 \times 3 \mathrm{~mm}^{2}$ area was reserved as alignment window while rest of the prism was coated with energy absorbing film, waterglass layer, reflective film and test film according to Fig. 1. Within the alignment window, the two orthogonal surfaces were left uncoated while the oblique surface was coated with a thin strip of aluminum film. A three-step procedure was followed for the purpose of laser alignment and for recording the data at point $B$ (Fig. 1). In the first step, the probe beam (532 nm crystal laser) of the Michelson interferometer was directed perpendicularly at the center of the window on test film side of the prism and reflected (by the oblique surface) out of the window on absorbing layer side. In the second step, a YAG sensitive paper was placed in front of the window on the absorbing layer side. The position of the $\mathrm{Nd}$ :YAG laser and the corresponding focusing lens was adjusted until the center of YAG spot (created by YAG firing) on the YAG sensitive paper overlapped with the
Fig. 4 Photograph of the experimental setup used for energy absorption study of polyurea films under laserinduced transient mixed-mode loading. The inset at the top left corner shows a fused silica prism of larger dimensions instead of actual specimen size $(3 \times 3 \times 20 \mathrm{~mm})$ for the demonstration purpose

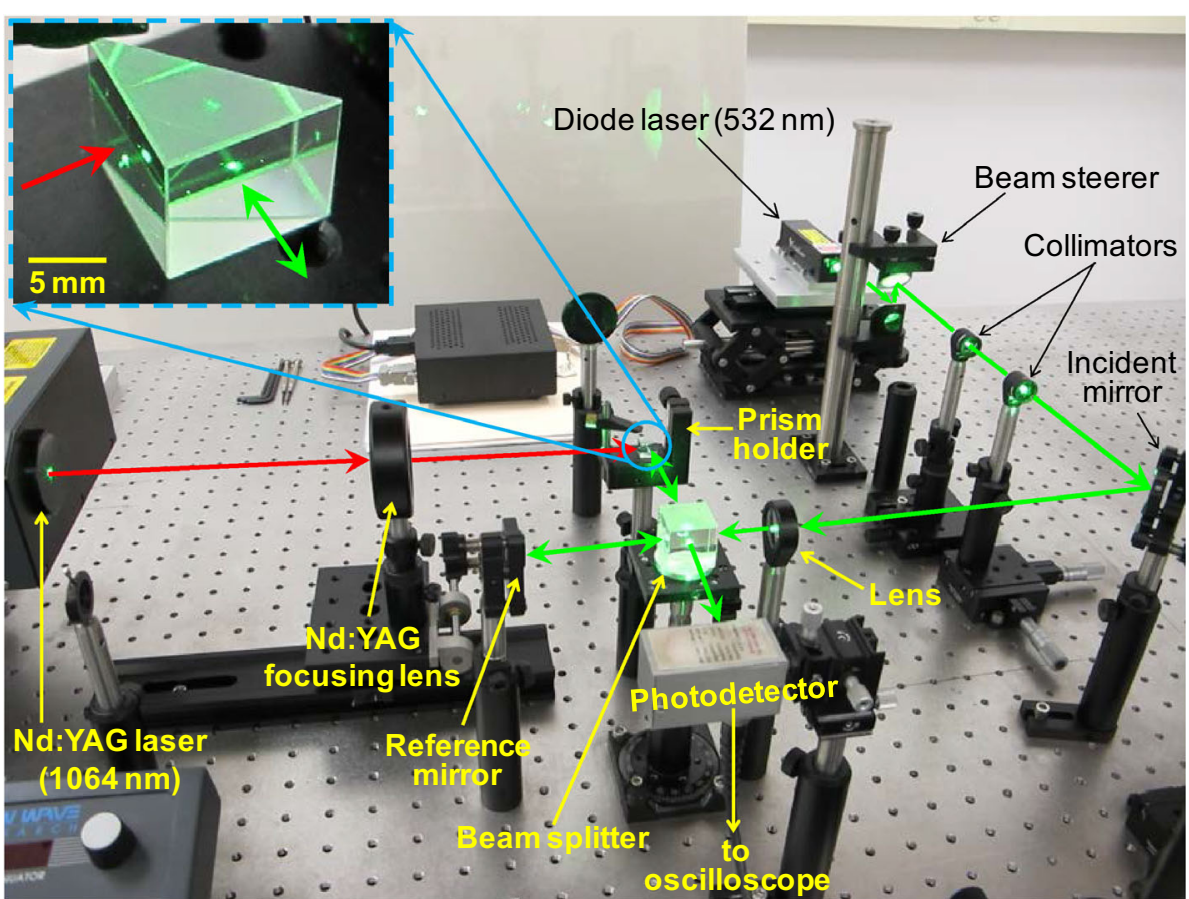




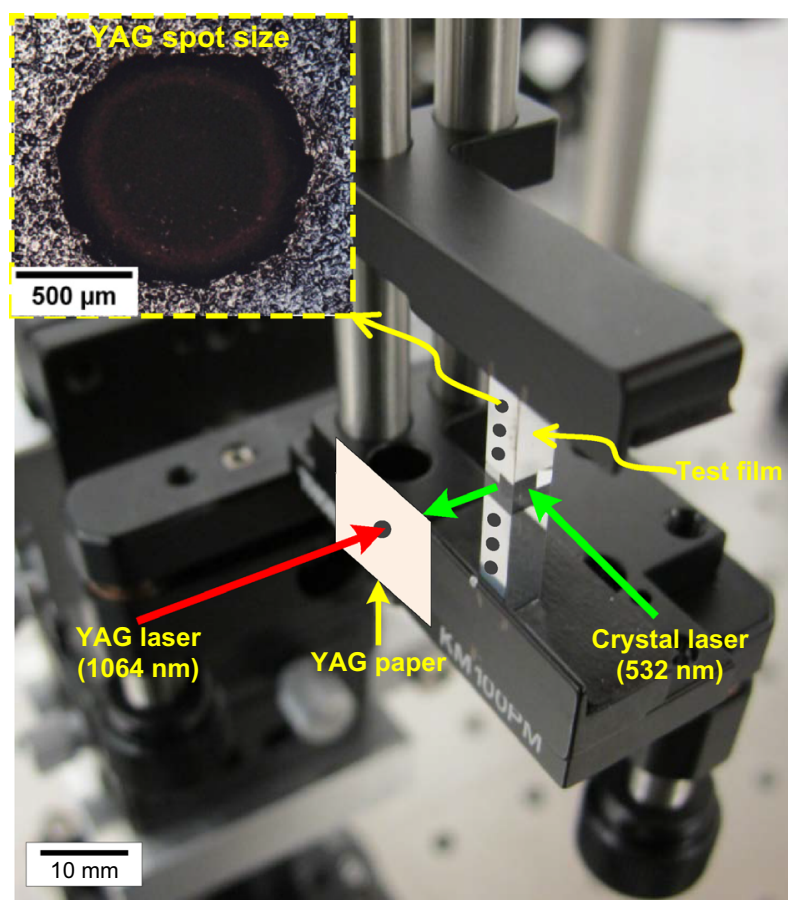

Fig. 5 Photograph showing the scheme of laser beam alignment. A right-angled fused silica prism $(3 \times 3 \times 20 \mathrm{~mm})$ sample is sitting on a prism mount attached to a 3-d micromechanical stage. The inset at the top left corner shows an optical micrograph depicting spot size $(\sim 1 \mathrm{~mm})$ of YAG impacts on the specimen

incoming probe beam as shown in Fig. 5. Once the alignment was completed, the relative position of both lasers was fixed. Next, in order to record surface deformation history at point $B$, the prism was translated horizontally by a pre-calculated distance of $0.5 \mathrm{~mm}$ (distance between points $A$ and $B$, for current geometry) relative to the position of probe beam. While testing, the specimen was moved vertically for multiple tests on either side of the uncoated region. In this work, the Nd:YAG laser was focused to a $1 \mathrm{~mm}$ diameter spot (inset of Fig. 5) on the energy absorbing layer.

\section{Interferometric Measurements and Fringe Analysis}

For the substrate geometry shown in Fig. $1, \theta_{1}\left(=45^{\circ}\right)$ and $\theta_{2}$ are the incident and reflected angles of $L_{1}$ and $S$ waves, respectively, and $\theta_{3}$ is the angle between the shear wave displacement (normal to the propagation direction of shear wave) and the test film. For the current sample dimensions, $\theta_{3}=\theta_{1}-\theta_{2}$, and the angle $\theta_{2}\left(=26.5^{\circ}\right)$ is given by Snell's law,

$\frac{\sin \theta_{1}}{\sin \theta_{2}}=\frac{C_{L}}{C_{S}}=k=1.584$,

where $C_{L}=5940 \mathrm{~m} / \mathrm{s}$ and $C_{S}=3750 \mathrm{~m} / \mathrm{s}$ are the longitudinal and shear wave speeds of the fused silica, respectively. According to linear wave propagation theory [37], the energy conservation of stress waves at the oblique surface is expressed,

$\left(\frac{u_{L_{2}}}{u_{L_{1}}}\right)^{2}+\left(\frac{u_{S}}{u_{L_{1}}}\right)^{2} \cdot \frac{\sin 2 \theta_{2}}{\sin 2 \theta_{1}}=1$,

where $u_{L_{1}}, u_{L_{2}}$ and $u_{\mathrm{s}}$ are displacement amplitudes of the stress waves $L_{1}, L_{2}$ and $S$, respectively. From elastic wave theory [38], the displacement amplitude ratios are given by,

$\frac{u_{L_{2}}}{u_{L_{1}}}=\frac{\sin 2 \theta_{1} \sin 2 \theta_{2}-k^{2} \cos ^{2} 2 \theta_{2}}{\sin 2 \theta_{1} \sin 2 \theta_{2}+k^{2} \cos ^{2} 2 \theta_{2}} \simeq-0.0645$,

$\frac{u_{S}}{u_{L_{1}}}=\frac{2 k \sin 2 \theta_{1} \cos 2 \theta_{2}}{\sin 2 \theta_{1} \sin 2 \theta_{2}+k^{2} \cos ^{2} 2 \theta_{2}} \simeq 1.1166$,

The negative output in Eq. (3) indicates that $L_{2}$ is reflected into a tensile stress wave. Using Eqs. (2)-(4), the energy $(E)$ distribution of $L_{2}$ and $S$ waves with respect to incident energy of $L_{1}$ wave, can be evaluated as,

$\frac{E_{L_{2}}}{E_{L_{1}}}=\left(\frac{u_{L_{2}}}{u_{L_{1}}}\right)^{2} \simeq 0.42 \%$ and
$\frac{E_{S}}{E_{L_{1}}}=\left(\frac{u_{S}}{u_{L_{1}}}\right)^{2} \cdot \frac{\sin 2 \theta_{2}}{\sin 2 \theta_{1}} \simeq 99.58 \%$.

More than $99 \%$ of the energy of the incident longitudinal wave, $L_{1}$, is mode-converted into the shear wave, $S$.

The interference intensity profile captured by the photodetector is reduced to a fringe count, $N(t)$, at a given time instant, $t$,

$I(t)=\frac{I_{\max }+I_{\min }}{2}+\frac{I_{\max }-I_{\min }}{2} \cdot \sin 2 \pi N(t)$,

where $I(t), I_{\max }$ and $I_{\min }$ are the photo-detector recorded light intensity signal, the intensity maximum and minimum, respectively. The fringe count, $N(t)$, of the interferogram is related to out-of-plane displacement, $u(t)$, and the optical wave length, $\lambda$, of the Michelson interferometer by,

$u_{n}(t)=\frac{\lambda}{2} \cdot N(t)$.

When the diagnostic beam of the Michelson interferometer is focused either at points $A, B$, or anywhere between $A$ and $B$ (Fig. 1), the interferometer detects both $L_{2}$ and $S$ waves. Although, the $L_{2}$ and $S$ regions are overlapped ${ }^{2}$, each wave can be easily distinguished based on the corresponding wave arrival times. However, the quality and the intensity of the wave signal vary depending on the location of the probe beam on the test film surface. The normal displacement $u_{L_{2}}$ can be obtained directly from the out-of-plane interferometric measurements at point $A$.

\footnotetext{
${ }^{2}$ For the substrate geometry in this work, the distance between points $A$ and $B$ is $0.5 \mathrm{~mm}$. For a $1 \mathrm{~mm}$ diameter YAG spot, the diameters of $L_{2}$ and $S$ waves fronts are $1 \mathrm{~mm}$ (centered at point $A$ ) and $1.33 \mathrm{~mm}$ (centered at point $B$ ), respectively [25].
} 
Subsequently, the shear displacement $u_{\mathrm{s}}$ is evaluated from $u_{L_{2}}$ using the mode-conversion coefficients from Eqs. (3) and (4), as shown in Eq. (8),

$\frac{u_{S}}{u_{L_{2}}}=\frac{2 k \sin 2 \theta_{1} \cos 2 \theta_{2}}{\sin 2 \theta_{1} \sin 2 \theta_{2}-k^{2} \cos ^{2} 2 \theta_{2}}$.

Alternatively, the shear displacement $u_{\mathrm{s}}$ can also be obtained from the shear part of the interferometric measurements at point $B$. Using any of the two approaches should yield the same results. In this work, the surface displacement history was inferred using the latter approach. Despite overlapping between the $L_{2}$ and the $S$ regions, the interferometric measurement at point $B$ offers a convenience of strong and easily discernible shear signal in the interferogram. In view of this, the probe beam of the Michelson interferometer has been directed at point $B$. Prior analyses by Wang et al. $[25,26]$ show that point $B$ is under mixed-mode loading and the out-of-plane displacement component $\left(u_{\perp}\right)$ at point $B$ is given by $u_{\perp}=u_{s} \beta$, where $\beta=0.778$.

In order to compute energy fluence $\left(\mathrm{J} / \mathrm{m}^{2}\right)$ development, the free surface velocity $v_{s}(t)$ profiles are obtained by numerical differentiation of surface displacement data,

$v_{s}(t)=\frac{d u_{s}(t) \beta}{d t}$.

The surface velocity is then converted to particle velocity $\left(v_{p}(t)=0.5 v_{s}(t)\right)$ [39] for energy flux calculations. The energy flux, $W(t)\left(\mathrm{J} /\left(\mathrm{m}^{2} \mathrm{~s}\right)\right)$, is evaluated from the velocity history using conservation of momentum and energy [40],

$W(t)=\frac{1}{2} \rho_{o}\left(v_{p}(t)\right)^{2} C_{S}$,

where $\rho_{\mathrm{o}}$ and $C_{s}$ are the initial density and the shear wave speed (see Table 1) of the substrate material, respectively. By performing numerical integration on energy flux over a given duration, the energy fluence histories, $E_{\mathrm{f}}\left(\mathrm{J} / \mathrm{m}^{2}\right)$ are obtained by,

$E_{f}=\int_{t_{1}}^{t_{2}} W(t) d t$.

\section{Results and Discussion}

Laser-induced dynamic mixed-mode loading experiments were performed on samples with aluminum and polyurea test films of thickness $100 \mathrm{~nm}$ and $2 \mu \mathrm{m}$, respectively. Initial benchmark experiments were carried out on $100 \mathrm{~nm}$ thick aluminum test films. The output fluence history from the benchmark tests on aluminum was used as the input fluence for polyurea films to estimate the energy absorption capacity of polyurea. A representative interferometric intensity signal as recorded by the photo-detector at point $B$ is shown in Fig. 6 for a $100 \mathrm{~nm}$ thick aluminum film tested at a laser fluence of $193 \mathrm{~mJ} / \mathrm{mm}^{2}$. As discussed earlier, due to the overlap between the $L_{2}$ and the $S$ regions, a longitudinal wave signal $L_{2}$ is also received at point $B$. Based on the wave speeds in fused silica, the geometry of the sample and the trigger delay of the YAG laser (100 ns delay between the Q-switch trigger and the firing of the YAG), the arrival times for the secondary longitudinal wave, $L_{2}$, and the shear wave, $S$, are evaluated theoretically and can be compared with the experimental observations as shown in Fig. 6. With reference to the trigger time of the Q-swtich of the YAG laser $(t=0 \mathrm{~ns})$, the arrival of the longitudinal wave $L_{2}$ is at about $t_{2}=607 \mathrm{~ns}$ when compared to the expected arrival time of $605 \mathrm{~ns}$ (100 ns trigger delay $+3 \mathrm{~mm}$ (travel distance)/ $\left.5940 \mathrm{~m} / \mathrm{s}\left(C_{L}\right)=605 \mathrm{~ns}\right)$. Likewise, the shear wave $S$ arrives at about $t_{3}=703 \mathrm{~ns}$, the expected arrival time (100 ns trigger delay $+3 \mathrm{~mm}$ (travel distance for $L_{2}$ wave)/ $5940 \mathrm{~m} / \mathrm{s}\left(C_{L}\right)+1.58 \mathrm{~mm}$ (travel distance for $S$ wave)/ $\left.3750 \mathrm{~m} / \mathrm{s}\left(C_{S}\right)=703 \mathrm{~ns}\right)$. Thus, the arrival times of both $L_{2}$ and $S$ stress waves are in good agreement with those expected from the theoretical calculations. Because of the negative nonlinear elasticity of fused silica, a shock wave appears at about $550 \mathrm{~ns}$ after the initially generated longitudinal stress pulse travels a short distance [30] in the substrate and the shock profile is maintained even after it is reflected from the oblique surface. At $t_{4}=792 \mathrm{~ns}$, a sharp turning point indicates the arrival of the decompression shock. In Fig. 6, the data also indicate the arrivals of low amplitude stresses at about $t_{1}=377 \mathrm{~ns}$. The presence of the low amplitude signals (indicated by the wide spacing)

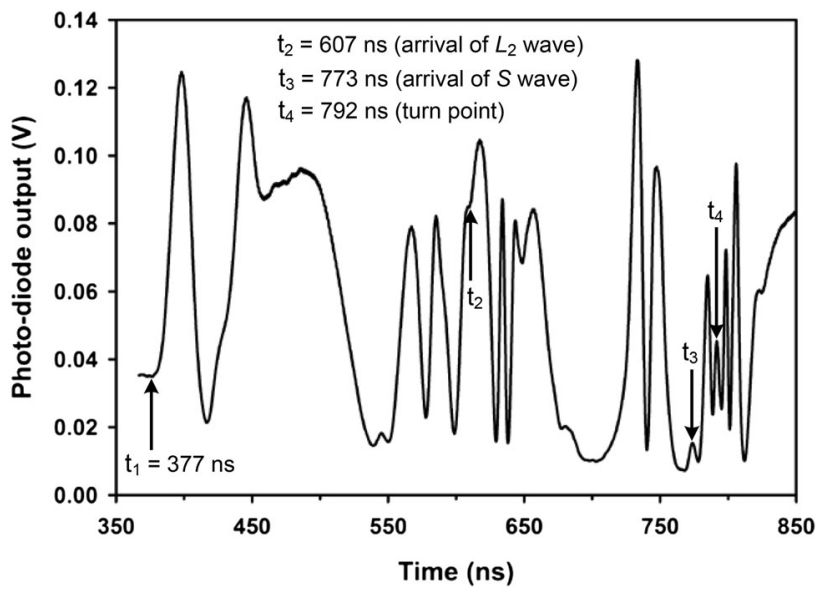

Fig. 6 Representative interferometric voltage signal detected at point $B$ (see Fig. 1) on a $100 \mathrm{~nm}$ thick aluminum film by the photo-detector in the Michelson interferometer of the dynamic mixed-mode experimental setup 
prior to the expected waves may be attributed to low amplitude longitudinal stress wave propagation diagonally from the sharp edges of the YAG impacted region [25].

Representative interferometric measurements are shown in Fig. 7 for a $100 \mathrm{~nm}$ thick aluminum film subjected to mixed-mode loading at a laser fluence of $193 \mathrm{~mJ} / \mathrm{mm}^{2}$. Fig. 7a shows a magnified view of the shear wave signal in the fringe data shown in Fig. 6. The time corresponding to the shear wave arrival $\left(t_{3}=773 \mathrm{~ns}\right)$ and the turning point $\left(t_{4}=792 \mathrm{~ns}\right)$ in the signal are indicated by arrows. The out-of-plane displacement history is calculated from the shear part of the signal, and is shown in Fig. $7 \mathrm{~b}$. The free surface velocity history shown in Fig. 7c is evaluated by numerical differentiation of the displacement profile. The surface velocity accelerates in a nearly monotonic fashion until it reaches a peak followed by a steep drop just before the turning point in the shear part of the signal. A noticeable departure from linearity of the velocity profile in the acceleration region is attributed to the noise introduced by
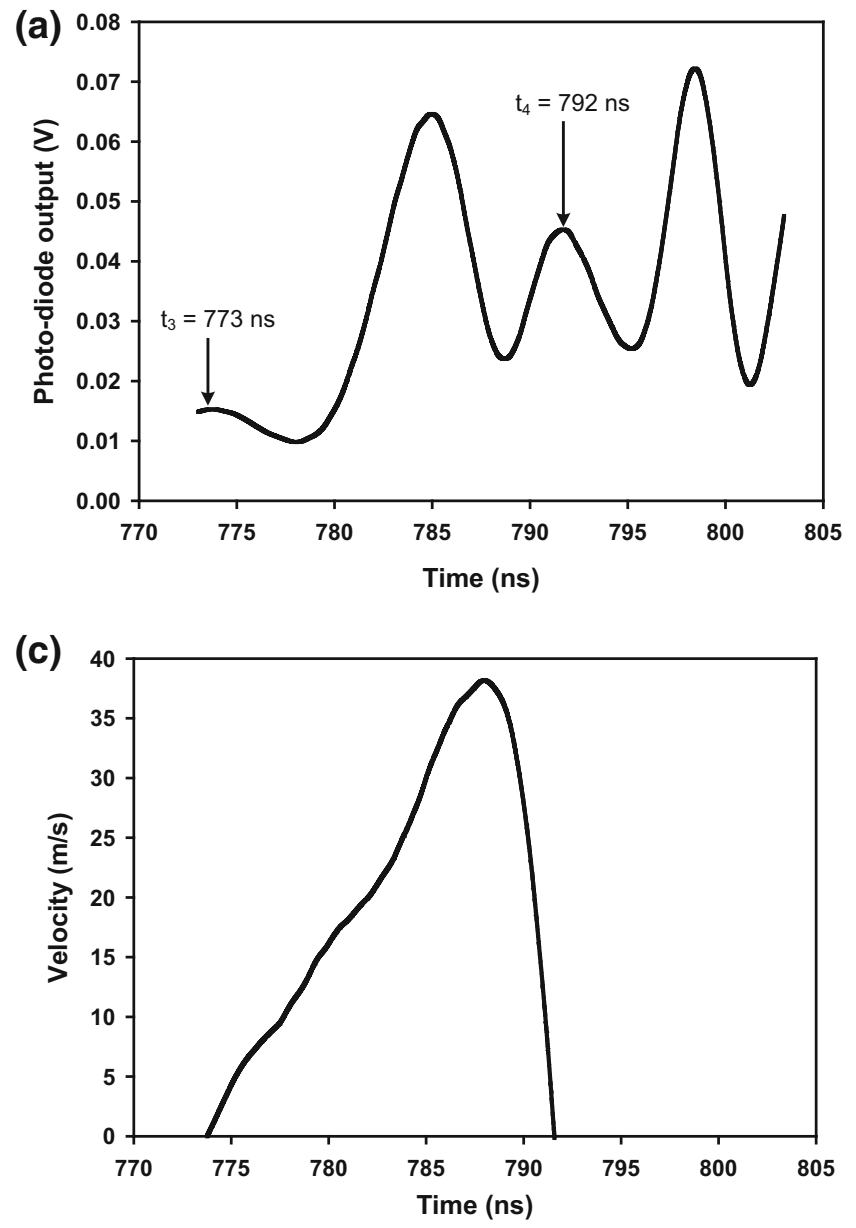

Fig. 7 Typical experimental data of a laser loading experiment on a $100 \mathrm{~nm}$ thick aluminum film at point $B$. a Expanded view of the shear wave signal in the fringe data of Fig. 7. b Corresponding free surface numerical derivation. The surface velocity is converted to particle velocity for energy flux calculations and by performing numerical integration on energy flux data over a given duration, the energy fluence is computed. The corresponding output energy fluence history is plotted in Fig. $7 \mathrm{~d}$ and is representative of the energy dissipation under mixed-mode loading.

Fig. 8a shows a typical interferometric voltage signal for a $2 \mu \mathrm{m}$ thick polyurea film captured at point $B$ at laser fluence of $193 \mathrm{~mJ} / \mathrm{mm}^{2}$. The arrival times for the $L_{2}\left(t_{2}\right)$ and $S\left(t_{3}\right)$ waves are computed for the polyurea film and are indicated by tilted arrows. A shock (sudden turning in the fringe signal) arrives at $792 \mathrm{~ns}$ as indicated by a vertical arrow. The voltage signal corresponding to shear region $\left(t_{2}\right.$ to $t_{3}$ ) is processed to obtain surface displacement, surface velocity and energy fluence histories. A systematic comparison is made between the interferometric measurements of aluminum and polyurea test films. The displacements profile, surface velocity and energy fluence histories for
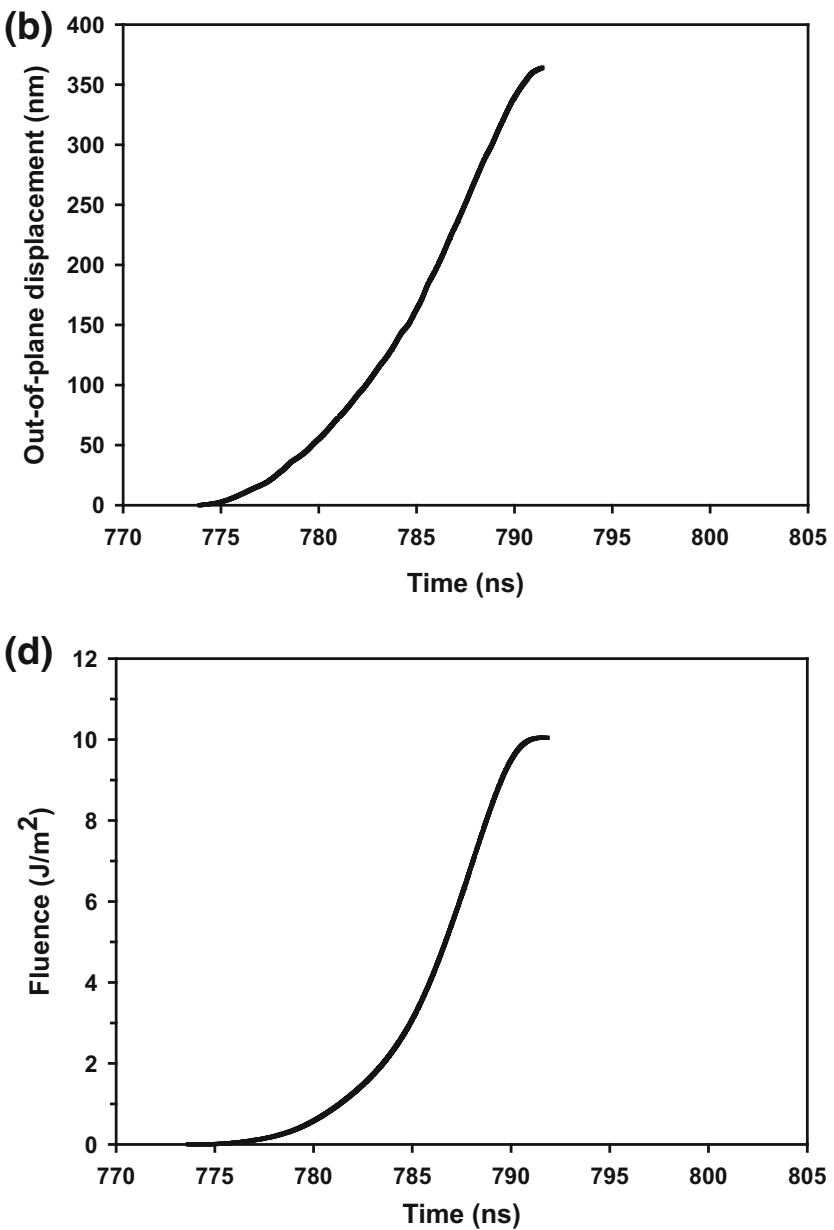

out-of-plane displacement history. c Free surface velocity history. d Output fluence evolution 

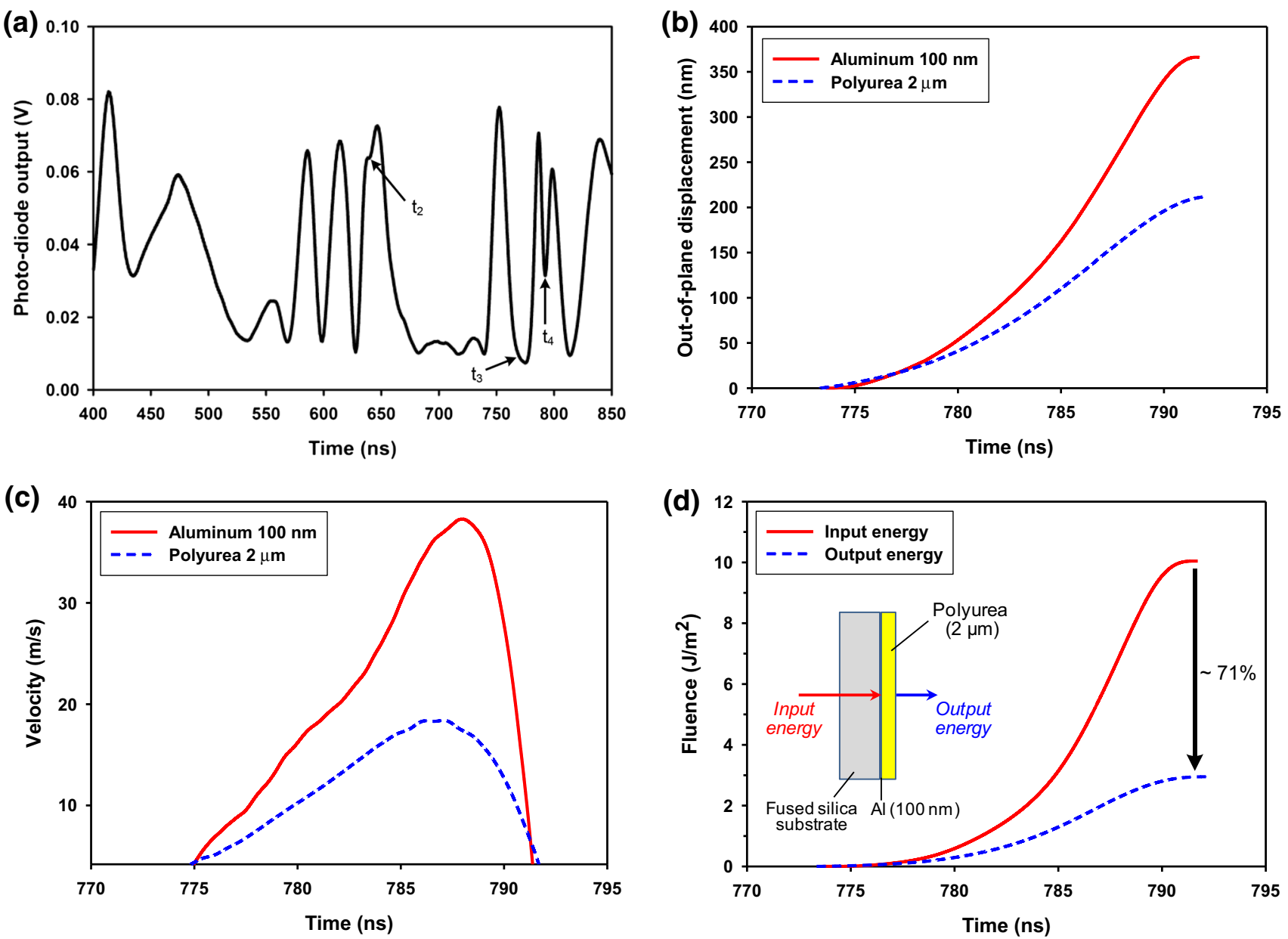

Fig. 8 a Typical interferometric fringe data captured at point $B$ (see Fig. 1) on a $2 \mu$ m thick polyurea film. b Comparison of free surface out-ofplane displacement histories. c Comparison of free surface velocity histories. d Comparison of input and output energy fluence histories

$100 \mathrm{~nm}$ thick aluminum and 2 um thick polyurea films are shown in Fig. 8b,c, and d, respectively. The out-of-plane surface displacement histories are compared in Fig. 8b. The displacement profiles show a monotonic increase in both cases, however, they differ in terms of the slopes and the peak values. As mentioned earlier, the polyurea films were fabricated on top of the $100 \mathrm{~nm}$ thick aluminum layer (see inset in Fig. 8d for film deposition scheme) pre-deposited on fused silica substrates, hence, the velocity or energy fluence from the shear measurements on aluminum film serve as input to the polyurea film. The surface velocity profiles in Fig. 8c are nearly Gaussian shape for both aluminum and polyurea films. In each case, the surface velocity increases monotonically until it attains a peak value followed by a deceleration. Due to the viscoelastic nature and higher film thickness, polyurea shows lower acceleration, low peak velocity and shallow deceleration when compared to the aluminum film. A $2 \mu \mathrm{m}$ thick polyurea film shows approximately $55 \%$ reduction in the peak velocity (input velocity $\sim 38 \mathrm{~m} / \mathrm{s}$ from the aluminum film to the polyurea film outputs to $\sim 17 \mathrm{~m} / \mathrm{s}$ ). The optically measured energy fluence histories for both films are compared in Fig. 8d. The input (from aluminum film) and output (polyurea film) energy fluence profiles show increasing trends with steep rise in the former relative to the latter. Upon comparing the peak values of input and output energy fluences, a $2 \mu \mathrm{m}$ thick polyurea film shows about $71 \%$ energy absorption under shock wave shear loading in a mixed-mode experimental setting.

The effect of laser fluence was studied on the energy absorption capability of $2 \mu \mathrm{m}$ thick polyurea thin films. A series of experiments were performed at laser fluences ranging from 157 to $217 \mathrm{~mJ} / \mathrm{mm}^{2}$ on $100 \mathrm{~nm}$ thick aluminum and $2 \mu \mathrm{m}$ thick polyurea thin films. With reference to the energy absorption scheme described in Fig. 8d, the input and output energies were compared to evaluate the percentage energy absorption by polyurea films. Figure 9 shows the variation of energy absorption (\%) for $2 \mu \mathrm{m}$ thick polyurea films tested over a range of laser fluences. Each data point represents an average of 3-4 tests and the error bars correspond to their standard deviation. The inset shows a photograph of the YAG impacted face of a shear 


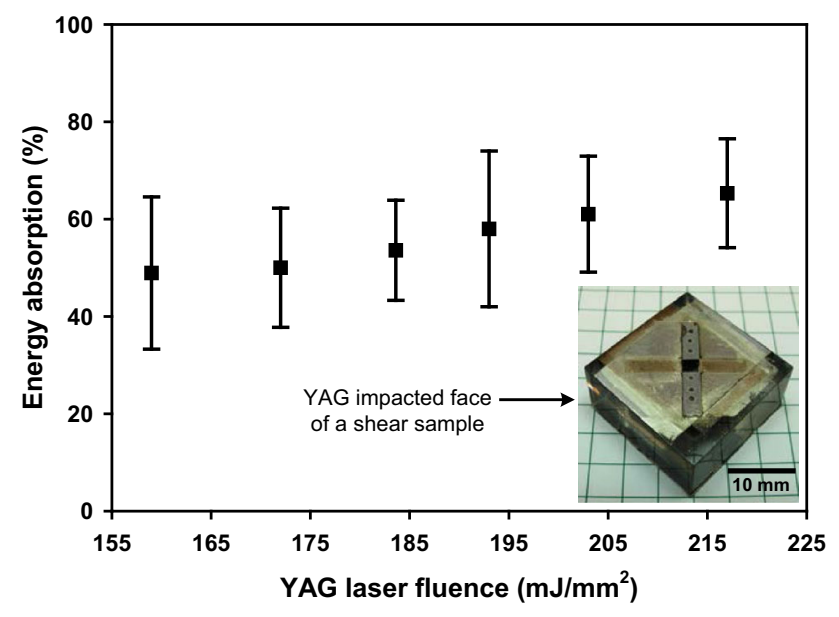

Fig. 9 Effect of laser fluence on energy absorption response of $2 \mu \mathrm{m}$ thick polyurea films. The inset shows a photograph of YAG impacted face of a shear test specimen

test specimen resting in a PDMS mold. The average energy absorption in Fig. 9 is approximately $50-55 \%$ at laser fluence ranging from 157 to $193 \mathrm{~mJ} / \mathrm{mm}^{2}$ followed by a modest increase of 60-65\% at higher laser fluence.

Polyurea is a micro-segregated amorphous phase with hard domains (aromatic, bulky, stiff, high $T_{\mathrm{g}}$ ) in a soft segment (aliphatic, long, flexible, low $T_{\mathrm{g}}$ ) viscoelastic
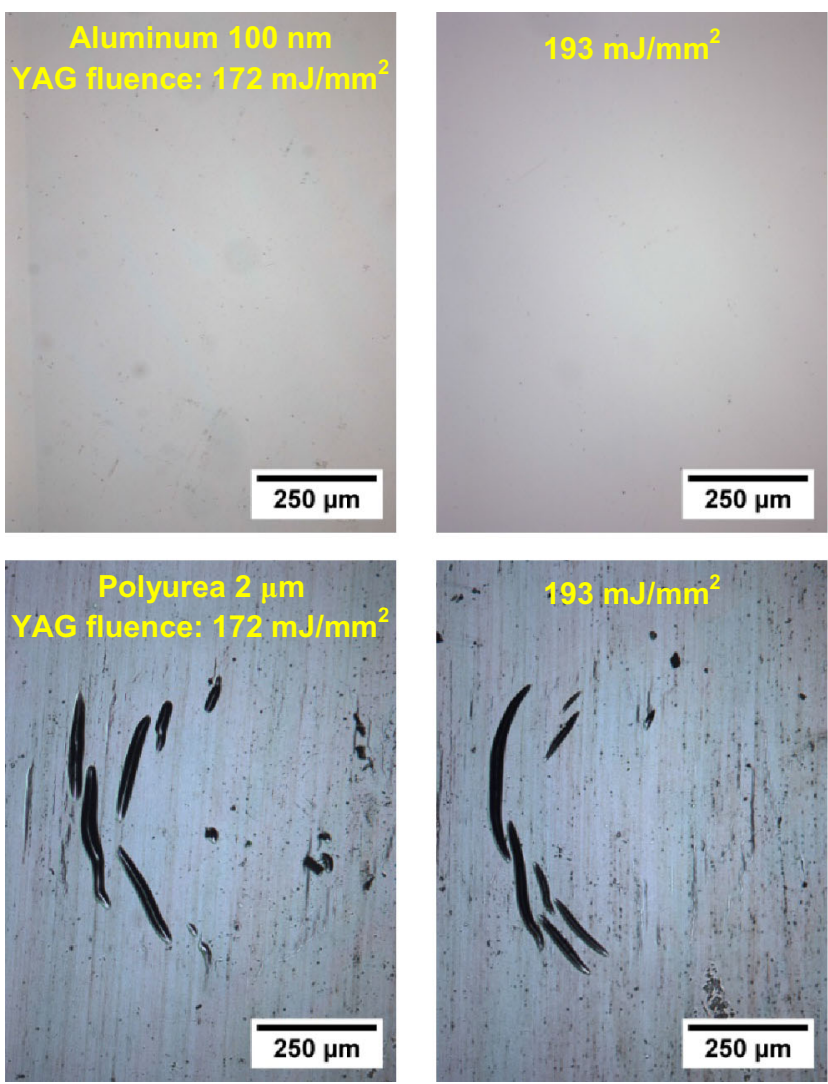

matrix. The combination of these hard and soft segments provides optimum strength and ductility to the polymer, as well as the transition of polyurea between its rubbery and glassy states at high strain rate loading results in inter-chain mobility, possibly responsible for its remarkable energy absorption characteristics. Furthermore, the propagating shock waves undergo multiple reflections because of the mechanical impedance mismatch in the specimen configuration adopted in this work, consisting of stiff (aluminum) and compliant (polyurea) films, and these reflections reduce the propagation velocity of shock waves by wave dispersions at the interface, and thereby enhance energy absorption.

The optical micrographs shown in Fig. 10 depict the effect of laser fluence on the deformation of thin films due to dynamic mixed-mode loading. A few representative images of damage patterns for the $100 \mathrm{~nm}$ thick aluminum and for the $2 \mu \mathrm{m}$ thick polyurea films are shown at varying laser fluence. The formation of wrinkles and tear marks indicate the presence of mode-mixity in the interfacial failure. For the case of $100 \mathrm{~nm}$ thick aluminum film at incident laser energies of 172 and $193 \mathrm{~mJ} / \mathrm{mm}^{2}$, no failure is visible, possibly due to the lack of threshold stress required to initiate the film deformation as well as due to the interfacial strengthening effect as the layer thickness
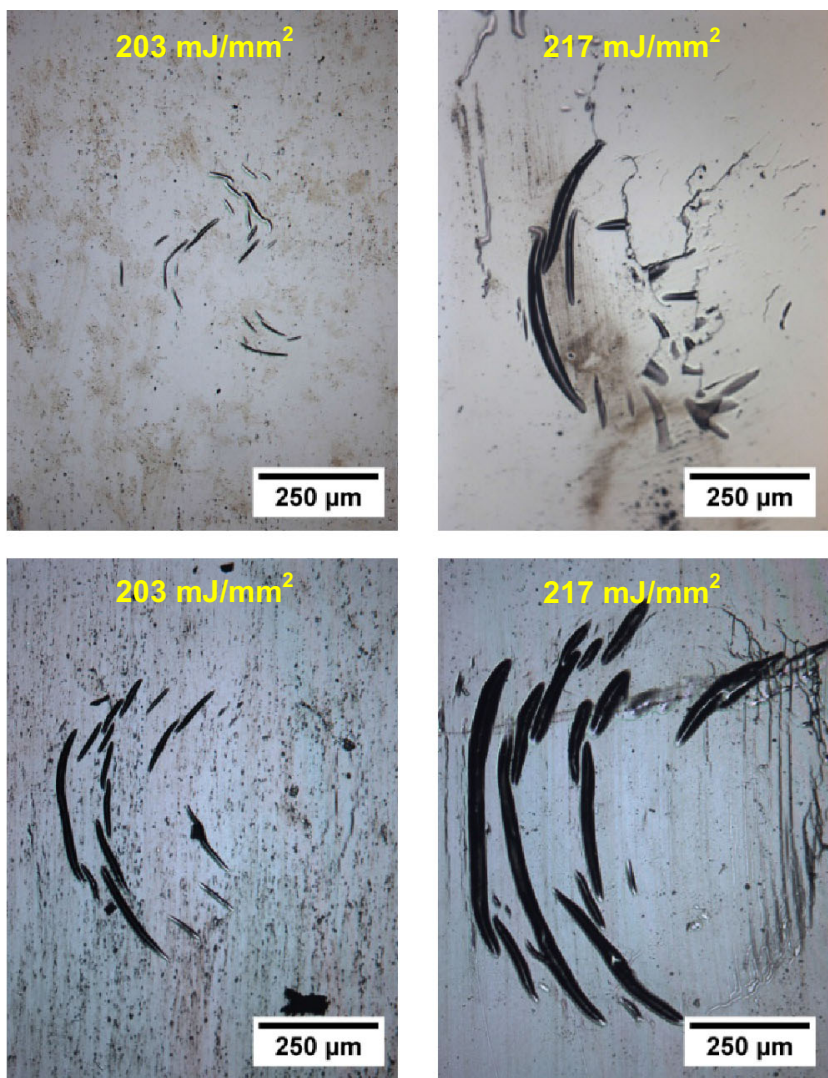

Fig. 10 Optical micrographs depicting the effect of laser fluence on the failure of $100 \mathrm{~nm}$ thick aluminum (top row) and $2 \mu \mathrm{m}$ thick polyurea (bottom row) films 
decreases from micron to nanometer scale [41]. After attaining threshold, the aluminum film shows interfacial damage at laser fluence of 203 and $217 \mathrm{~mJ} / \mathrm{mm}^{2}$, however, the damage zone is relatively smaller at $203 \mathrm{~mJ} / \mathrm{mm}^{2}$, than that at $217 \mathrm{~mJ} / \mathrm{mm}^{2}$. In the case of $2 \mu \mathrm{m}$ thick polyurea film, damage increases with increasing laser fluence. More significant wrinkling and several tear marks occur in both aluminum and polyurea films at the highest laser fluence $217 \mathrm{~mJ} / \mathrm{mm}^{2}$. Note that the film wrinkling is attributed to the accumulation of in-plane shear motion from longitudinal to shearing direction (from right to left in the micrographs, see Fig. 1, $B \leftarrow A$ ). Furthermore, the density and size of wrinkles increases along the shearing direction with increasing laser fluence. The extent of damage and large size of wrinkles in polyurea is possibly due to the higher film thickness $(2 \mu \mathrm{m})$ as compared to the aluminum film $(100 \mathrm{~nm})$. In both films, the formation of wrinkles and their direction of deformation indicates shear as the dominant failure mode.

\section{Conclusions}

The energy absorption behavior of thin poylurea films $(2 \mu \mathrm{m})$ was studied under dynamic shear loading using a laser-induced mixed-mode experimental setup. Mode mixity at the film was induced by directing mode-converted shear and longitudinal stress waves from the oblique surface of a fused silica substrate. The interferometric fringe data revealed the development of a shock due to nonlinear elastic characteristics of fused silica substrate. Test results indicated significant reduction in the peak surface velocity by polyurea films relative to the input velocity. A $2 \mu \mathrm{m}$ thick polyurea film showed approximately 50-65\% energy absorption at varying laser fluences under shock wave shear loading. The wrinkling and tearing patterns of failed films were consistent with mixedmode loading, and their directions of failure demonstrated shear as the dominant failure mode.

Acknowledgments The authors would like to thank the office of Naval Research for supporting this research through a grant ONR MURI N00014-12-1-0828. The use of e-beam instruments in the Frederick Seitz Materials Research Laboratory Central Facilities, University of Illinois is also gratefully acknowledged.

\section{References}

1. Yi J, Boyce MC, Lee GF, Balizer E (2006) Large deformation rate-dependent stress-strain behavior of polyurea and polyurethanes. Polymer 47(1):319-329

2. Das S, Yilgor I, Yilgor E, Inci B, Tezgel O, Beyer FL, Wilkes GL (2007) Structure-property relationships and melt rheology of segmented, non-chain extended polyureas: effect of soft segment molecular weight. Polymer 48(1):290-301
3. Fragiadakis D, Gamache R, Bogoslovov RB, Roland CM (2010) Segmental dynamics of polyurea: effect of stoichiometry. Polymer 51(1):178-184

4. Qiao J, Amirkhizi AV, Schaaf K, Nemat-Nasser S, Wu G (2011) Dynamic mechanical and ultrasonic properties of polyurea. Mech Mater 43(10):598-607

5. Mock W, Bartyczak S, Lee G, Fedderly J, Jordan K (2009) Dynamic properties of polyurea 1000. AIP Conference Proceedings 1195 (1):1241-1244

6. Roland CM, Twigg JN, Vu Y, Mott PH (2007) High strain rate mechanical behavior of polyurea. Polymer 48(2):574-578

7. Sarva SS, Deschanel S, Boyce MC, Chen W (2007) Stress-strain behavior of a polyurea and a polyurethane from low to high strain rates. Polymer 48(8):2208-2213

8. Shim J, Mohr D (2009) Using split Hopkinson pressure bars to perform large strain compression tests on polyurea at low, intermediate and high strain rates. Int $J$ Impact Eng 36(9):1116-1127

9. Tekalur SA, Shukla A, Shivakumar K (2008) Blast resistance of polyurea based layered composite materials. Compos Struct 84(3):271-281

10. Gardner N, Wang E, Kumar P, Shukla A (2012) Blast mitigation in a sandwich composite using graded core and polyurea interlayer. Exp Mech 52(2):119-133

11. Youssef G, Gupta V (2011) Dynamic response of polyurea subjected to nanosecond rise-time stress waves. Mech Time Depend Mater 16(3):317-328

12. Amini MR, Isaacs J, Nemat-Nasser S (2010) Investigation of effect of polyurea on response of steel plates to impulsive loads in direct pressure-pulse experiments. Mech Mater 42(6):628-639

13. Roland CM, Fragiadakis D, Gamache RM (2010) Elastomersteel laminate armor. Compos Struct 92(5):1059-1064

14. Xue L, Mock W Jr, Belytschko T (2010) Penetration of DH-36 steel plates with and without polyurea coating. Mech Mater 42(11):981-1003

15. Jajam KC, Bird SA, Auad ML, Tippur HV (2013) Tensile, fracture and impact behavior of transparent Interpenetrating Polymer Networks with polyurethane-poly(methyl methacrylate). Polym Test 32(5):889-900

16. Jajam KC, Tippur HV, Bird SA, Auad ML (2014) Dynamic fracture and impact energy absorption characteristics of PMMAPU transparent interpenetrating polymer networks (IPNs). In: Song B, Casem D, Kimberley J (eds) Dynamic behavior of materials. Conference proceedings of the society for experimental mechanics series, vol 1. Springer International Publishing, pp 277-284

17. Bahei-El-Din YA, Dvorak GJ (2007) Behavior of sandwich plates reinforced with polyurethane/polyurea interlayers under blast loads. J Sandw Struct Mater 9(3):261-281

18. Grujicic M, Bell WC, Pandurangan B, He T (2010) Blast-wave impact-mitigation capability of polyurea when used as helmet suspension-pad material. Mater Des 31(9):4050-4065

19. Grujicic M, Yavari R, Snipes JS, Ramaswami S, Runt J, Tarter J, Dillon G (2012) Molecular-level computational investigation of shock-wave mitigation capability of polyurea. J Mater Sci 47(23):8197-8215

20. Moshe E, Eliezer S, Dekel E, Ludmirsky A, Henis Z, Werdiger M, Goldberg IB, Eliaz N, Eliezer D (1998) An increase of the spall strength in aluminum, copper, and Metglas at strain rates larger than $10^{7} \mathrm{~s}^{-1}$. J Appl Phys 83(8):4004-4011

21. Eliezer S, Gilath I, Bar-Noy T (1990) Laser-induced spall in metals: experiment and simulation. J Appl Phys 67(2):715-724

22. Fortov VE, Kostin VV, Eliezer S (1991) Spallation of metals under laser irradiation. J Appl Phys 70(8):4524-4531

23. Yang LC (1974) Stress waves generated in thin metallic films by a Q-switched ruby laser. J Appl Phys 45(6):2601-2608 
24. Yuan J, Gupta V (1993) Measurement of interface strength by the modified laser spallation technique. I. Experiment and simulation of the spallation process. J Appl Phys 74(4):2388-2396

25. Wang J, Sottos NR, Weaver RL (2003) Mixed-mode failure of thin films using laser-generated shear waves. Exp Mech 43(3):323-330

26. Wang J, Sottos NR, Weaver L (2004) Tensile and mixed-mode strength of a thin film-substrate interface under laser induced pulse loading. J Mech Phys Solids 52(5):999-1022

27. Kitey R, Geubelle PH, Sottos NR (2009) Mixed-mode interfacial adhesive strength of a thin film on an anisotropic substrate. J Mech Phys Solids 57(1):51-66

28. Hu L, Wang J (2006) Pure-shear failure of thin films by laserinduced shear waves. Exp Mech 46(5):637-645

29. Evans AG, Hutchinson JW (1995) The thermomechanical integrity of thin films and multilayers. Acta Metall Mater 43(7):2507-2530

30. Wang J, Weaver RL, Sottos NR (2003) Laser-induced decompression shock development in fused silica. J Appl Phys 93(12):9529-9536

31. Gupta V, Kireev V, Tian J, Yoshida H, Akahoshi H (2003) Glassmodified stress waves for adhesion measurement of ultra thin films for device applications. J Mech Phys Solids 51(8):1395-1412

32. Wang J, Weaver R, Sottos N (2002) A parametric study of laser induced thin film spallation. Exp Mech 42(1):74-83

33. Gupta V, Yuan J, Pronin A (1993) Nanosecond rise-time laserproduced stress pulses with no asymptotic decay. Rev Sci Instrum 64(6):1611-1613
34. Yuan J, Gupta V, Pronin A (1993) Measurement of interface strength by the modified laser spallation technique. III. Experimental optimization of the stress pulse. J Appl Phys 74(4):2405-2410

35. Neubauer WG, Vogt RH, Dragonette LR (1974) Acoustic reflection from elastic spheres. I. Steady-state signals. J Acoust Soc Am 55(6):1123-1129

36. Bennett HE, Porteus JO (1961) Relation between surface roughness and specular reflectance at normal incidence. J Opt Soc Am 51(2):123-129

37. Miklowitz J (1978) The theory of elastic waves and waveguides. Applied mathematics and mechanics, vol vol 22. North-Holland Publishing Company, New York

38. Graff KF (1991) Wave motion in elastic solids. Dover Publications, New York

39. Luo S-N, Han L-B, Xie Y, An Q, Zheng L, Xia K (2008) The relation between shock-state particle velocity and free surface velocity: a molecular dynamics study on single crystal $\mathrm{Cu}$ and silica glass. J Appl Phys 103(9):093530

40. Forbes JW (2012) Shock wave compression of condensed matter. Springer-Verlag, Berlin

41. Misra A, Hirth JP, Hoagland RG (2005) Length-scale-dependent deformation mechanisms in incoherent metallic multilayered composites. Acta Mater 53(18):4817-4824 